\title{
Examining the relationships between emotional disorder symptoms in a mixed sample of community adults and patients: A network analysis perspective
}

\author{
A. De la Rosa-Cáceres ${ }^{1,2} \cdot$ P. Sayans-Jiménez ${ }^{3} \cdot$ S. Stasik-O'Brien ${ }^{4} \cdot$ M. Sanchez-Garcia ${ }^{1,2} \cdot$ F. Fernández-Calderón ${ }^{1,2}$. \\ Carmen Díaz-Batanero ${ }^{1,2}$ (1)
}

Accepted: 11 February 2022

(C) The Author(s) 2022

\begin{abstract}
According to the literature, comorbidity rates observed on emotional disorders are linked to how the main diagnostic classification systems have traditionally defined these disorders. This paper aims to analyze the structure of symptoms evaluated with the Inventory of Depression and Anxiety Symptoms-II (IDAS-II) with network analysis. A mixed sample $(n=2021)$ of 1692 community adults and 329 patients was used. 14.79\% $(n=299)$ of the sample met the diagnostic criteria for at least one DSM-5 mental disorder and 5.29\% $(n=107)$ had diagnostic comorbidity. The sample was randomly divided into two sub-samples: estimation sample $(n=1010)$ and replication sample $(n=1011)$. The detection of community structures was carried out on estimation sample using the walktrap algorithm. Four local inference measures were estimated: Strength, onestep Expected Influence, two-step Expected Influence, and node predictability. Exploratory graphic analysis of modularity yielded an optimal solution of two communities on estimation sample: first linked to symptoms of depression and anxiety and second grouping symptoms of bipolar disorder and obsessive - compulsive disorder. Mania, Panic, Claustrophobia, and Low Well-Being Bridge emerged as bridge symptoms, connecting the two substructures. Networks estimated on replication subsamples did not differ significantly in structure. Dysphoria, Traumatic Intrusions and Checking and Ordering were the symptoms with greatest number of connections with rest of the network. Results sheds light on specific links between emotional disorder symptoms and provides useful information for the development of transdiagnostic interventions by identifying the influential symptoms within the internalizing spectrum.
\end{abstract}

Keywords Emotional disorders · Comorbidity · IDAS-II · HiTOP · Transdiagnostic · Network analysis

\section{Introduction}

Depression and anxiety affect $4.4 \%$ and $3.6 \%$ of the world's population respectively, having serious consequences for health, quality of life and severe negative

Carmen Díaz-Batanero

carmen.diaz@dpsi.uhu.es

1 Department of Clinical and Experimental Psychology, University of Huelva, Huelva, Spain

2 Research Center for Natural Resources, Health and the Environment, University of Huelva, Huelva, Spain

3 Department of Psychology, University of Almería, Almería, Spain

4 Department of Psychology, Knox College, Galesbug, IL, USA clinical outcomes such as suicidal behavior (World Health Organization, 2017). Although previous studies have identified neurobiological factors that explain the complexity of suicide (Orsolini et al., 2020) and provide advances in treatments (De Berardis et al., 2018), suicidal behavior still remains a real challenge associated with mental disorders comorbidity (Quevedo et al., 2020). According to empirical literature (e.g., Skodol, 2012), high rates of co-morbidity are linked to the way in which diagnostic classification systems have traditionally defined disorders. These systems, which use polythetic criteria, establish categorical classifications according to an observed set of symptoms, resulting in patients with different symptoms receiving the same diagnosis (Skodol, 2012).

In order to overcome the limitations of this taxonomic approach, transdiagnostic proposals look beyond the traditional classification schemes (Barlow et al., 2017; Kotov 
et al., 2017). The translation of this conceptualization into the applied field has allowed for the development of unified intervention protocols, yielding more effective and cheaper interventions (Barlow et al., 2017). Whilst these are promising findings, the transdiagnostic approach still requires a better understanding of the relationships between symptoms.

Past decade, empirical network models have provided a complementary approach to understanding the interaction between symptoms (Cramer et al., 2010). The analysis of bridge symptoms connecting different substructures offers a complementary view of the comorbidity phenomenon (Jones et al., 2019). At the same time, network analysis allows us to analyze the influence that each symptom has on the other symptoms in a network (Robinaugh et al., 2016), identifying which will have the greatest potential to influence the overall network (Borsboom, 2017). This information could then be useful for designing transdiagnostic interventions.

Network analysis has already been applied to the study of comorbid emotional disorders, with interesting results (see review by Contreras et al. (2019)). Overall, a high connectivity between depression and anxiety symptoms has been observed (Bekhuis et al., 2016; McElroy et al., 2018). The symptoms of post-traumatic stress disorder (PTSD) are, however, shown in a different cluster, and are connected to the depression cluster by the symptoms of "irritability" or "sleep problems" (Afzali et al., 2017; Choi et al., 2017). McNally et al. (2017) have also shown the lack of connection between the symptoms linked to depression and the symptoms of obsessive-compulsive disorder (OCD), which appear solely connected through "sadness" but not through sleep problems. The results of previous work have also identified dysphoria and lassitude to be the most influential symptoms in the networks obtained for depression (e.g., Funkhouser et al., 2020), whilst traumatic intrusions are the most influential for PTSD (Contractor et al., 2020; Gilbar, 2020) and the checking and ordering symptoms for OCD networks (Zhang et al., 2019).

Whilst these results are of interest, to our knowledge, studies of this type use different instruments to assess each group of symptoms (e.g. Choi et al., 2017), involving different instructions and time frames for evaluation (Watson et al., 2012). This affect measurement and network estimation (Bekhuis et al., 2016). Only Afzali et al. (2017) explored the relationship between major depressive disorder and PTSD with the same instrument (the ICD) although it provides a categorical measure of the evaluated symptomatology.

In this regard, the Inventory of Depression and Anxiety Symptoms (IDAS; Watson et al., 2007) and the IDAS-II (Watson et al., 2012) have been identified as effective tools for measuring several symptoms and spectra of the transdiagnostic models of psychopathology (Kotov et al., 2017).
Funkhouser et al. (2020), found consistent connections between the symptoms of dysphoria and lassitude, as well as between dysphoria and social anxiety, evaluated with the IDAS, although this work did not specifically aim to analyze the bridge symptoms that could explain comorbidity. The analysis of the structure of IDAS-II could further provide a broader perspective on the configuration of the relationships between emotional disorders, as it additionally measures symptoms of bipolar disorder (BD) and OCD as well as contains more expansive coverage of PTSD and social anxiety symptoms.

Given the above, this work examines, through empirical networks: 1) the structure of the relationships between the dimensional symptoms involved in the emotional disorders evaluated with the IDAS-II; 2) the bridge symptoms between the identified substructures; and 3) the influence that each symptom has on the rest of the network. According to previous studies we expected that: 1) the symptoms of depression and anxiety will be highly connected in a cluster that, in turn, will show less connections with the symptoms of $\mathrm{BD}$ and $\mathrm{OCD} ; 2)$ the symptoms acting as a bridge between these will be related to irritability and sadness; and 3) the symptoms with the strongest influence will be dysphoria for depression, traumatic intrusions for PTSD, and checking and ordering for OCD.

\section{Material and Methods}

\section{Participants}

In order to increase the variability of the scores and thus represent the dimensional continuum from normality to pathology, the study was conducted on a mixed sample of 2021 persons, composed of both adults from the community population $(n=1692)$ and patients $(n=329)$.

The community adults were recruited in two phases of data collection: a) Wave 1, 620 adults selected by means of non-probability sampling in the province of Huelva (Spain); and b) Wave 2, 1,072 adults recruited by stratified random sampling, proportionally represented in the Spanish population according to age group, sex, and geographic area. The equivalence between both samples was verified. Inclusion criteria on community sample was being between 18 and 80 years old and not having a diagnose on any mental disorder.

The patient sample consisted of 329 patients from public and private mental health services in the province of Huelva (Spain). Inclusion criteria on clinical sample was being between 18 and 80 years old and being under treatment in a mental health service during the data collection. Those who met any of the following characteristics were excluded from both 
samples (community adults and patients): having been diagnosed with a medical or psychological disorder that disqualified them from taking the tests, or not signing the informed consent form.

Of the total number of participants $(n=2,021)$, $51.6 \%$ were women, and aged between 18 and 80 years $(M=42.90 ; S D=14.64) .1 .8 \%$ had not completed primary education, $8.5 \%$ had completed primary education, $16.5 \%$ had completed secondary education, $34.3 \%$ had completed post-compulsory education, $37.6 \%$ had completed university studies, and $1.2 \%$ had completed a university doctorate. With regard to employment status, $55.6 \%$ were working. $14.79 \%(n=299)$ of the sample met the diagnostic criteria for at least mental one disorder mental disorder according to Diagnostic and Statistical Manual of Mental Disorders, fifth edition (DSM-5) and $5.29 \%(n=107)$ had diagnostic comorbidity. Table 1 shows the diagnoses present in the sample. The most frequent diagnostic categories were Depressive Disorders (5.84\%) and Anxiety Disorders (5.10\%).

\section{Measures}

The IDAS-II (Watson et al., 2012) was administered in its Spanish version (De la Rosa-Cáceres et al., 2020). The 99 items with a Likert response format from 1 ("not at all") to 5 ("extremely") are grouped in 18 specific scales. Participants scored the severity of their symptoms during the last two weeks, where higher scores indicate greater severity of symptoms. In the present study, this instrument was found to be internally consistent (Cronbach's alpha $=0.91)$. Internal consistency of all subscales are presented in Table 2.
In addition, questions regarding sociodemographic variables related to sex, age, educational level, employment, and marital status were included.

\section{Procedure}

Community adults from wave 2 completed the IDAS-II in an online format, whilst a psychologist administered the Spanish version of IDAS-II in paper and pencil format to the rest of the community and patient samples $(n=949)$. The latter completed the IDAS-II in rooms set up in the centers where they were recruited. All participants were informed about the anonymous and voluntary nature of their participation in the study and gave their written informed consent which remained in the custody of the research group. This study has the approval of the Bioethics Committee of Biomedical Research of Andalusia (Spain) (file number PI 040/18).

\section{Data Analysis}

Results revealed the absence of multivariate normality for asymmetry (Mardia $=8092.19$ ) and kurtosis (Mardia $=89.62$ ). The estimation of the network and its properties was conducted using the Graphical Least Absolute Shrinkage and Selection Operator (GLASSO; Friedman et al., 2008) algorithm in combination with the Extended Bayesian Information Criterion (EBIC; Chen \& Chen, 2008) selection model applied to the nonparanormal transformation (Liu et al., 2009) of the data set. The EBIC hyperparameter $\gamma$ was set at 0.5 . The arrangement of the nodes was established using the Fruchterman-Reingold algorithm (Fruchterman \& Reingold, 1991).
Table 1 DSM-5 Diagnoses distribution

\begin{tabular}{llll}
\hline & $n$ & $\begin{array}{l}\text { \% over patients } \\
(n=329)\end{array}$ & $\begin{array}{l}\text { \% over com- } \\
\text { plete sample } \\
(n=2021)\end{array}$ \\
\hline Neurodevelopmental Disorders & & 7.60 & 1.24 \\
Schizophrenia Spectrum and Other Psychotic Disorders & 23 & 6.99 & 1.14 \\
Bipolar and Related Disorders & 12 & 3.65 & 0.59 \\
Depressive Disorders & 118 & 35.87 & 5.84 \\
Anxiety Disorders & 103 & 31.31 & 5.10 \\
Obsessive-Compulsive and Related Disorders & 9 & 2.74 & 0.44 \\
Trauma- and Stressor-Related Disorders & 77 & 23.40 & 3.80 \\
Dissociative Disorders & 2 & 0.61 & 0.10 \\
Somatic Symptom and Related Disorders & 2 & 0.61 & 0.10 \\
Feeding and Eating Disorders & 6 & 1.82 & 0.29 \\
Disruptive, Impulse-Control, and Conduct Disorders & 5 & 1.52 & 0.25 \\
Substance-Related and Addictive Disorders & 11 & 3.34 & 0.54 \\
Personality Disorders & 30 & 9.12 & 1.48 \\
Do not meet diagnostic criteria & 30 & 9.12 & 1.48 \\
\hline
\end{tabular}


The selected sample was randomly divided into two subsamples: estimation sample $(n=1010)$ and replication sample $(n=1011)$. The sample size of the estimation sample $(n=1010)$ was adequate for the estimation of the network based on simulation analyses (Epskamp et al., 2018) (see Online Resource 1). The detection of community structures was carried out on estimation sample using the walktrap algorithm (Pons \& Latapy, 2005) and the optimal number of steps was set using the highest cluster's modularity. The modularity index resulting from the exploratory graphic solution was compared with obtained of the three-factor structure presented in De la Rosa-Cáceres et al. (De la RosaCáceres et al., 2020).

Four local inference measures were estimated: Strength, one-step Expected Influence (EI1), twostep Expected Influence (EI2), and node predictability Haslbeck \& Waldorp, 2018; Robinaugh et al., 2016). Strength and EI are relative measures of centrality that provide information about the relationships between each node and the rest of the network. EI2 takes into account, besides, the relations with the nodes that are of an indirect nature (Robinaugh et al., 2016). Further, the predictability of the node is an absolute measurement of interconnection that indicates the proportion of variance of each node that is explained by its neighboring nodes (Haslbeck \& Waldorp, 2018).

Participation Coefficient (PC) and Participation Ratio (PR) (Letina et al., 2019) were applied to analyze the heterogeneity of the network nodes. The PC analyzes how the nodes distribute their edges among the different communities, where higher values of PC indicate that the nodes distribute their edges more equally among the network communities. The PR takes into account both the number and strength of the edges connected to each node, so that higher values of PR are indicative of nodes with more numerous and stronger edges. The PC and PR values were transformed to the same scale of values between 0 and 1 to facilitate their interpretation.

To increase the understanding of comorbidity, three bridge centrality indices were estimated (Jones, 2020; Jones et al., 2019): bridge strength, which indicates the total connectivity of the node with nodes of other communities with which it is directly related; bridge EI1, which indicates the general increase in activation of the node with nodes of other communities with which it is directly related; and bridge EI2, which, like bridge EI1, indicates the general increase in the activation of other communities, but in this case by also taking into account the effect on nodes of other communities with which it is indirectly related. Finally, bridge nodes/ symptoms were identified by applying a blind cut-off point at the 80th percentile of bridge strength (Jones et al., 2019).

The precision of the estimated relationships in the network was estimated by calculating the values of the edge weights with a $95 \%$ confidence interval (CI) from a nonparametric bootstrap with subsamples repeated 1,000 times (Epskamp et al., 2018). The stability of the network centrality indices was evaluated by means of a person-dropping bootstrap procedure that provides a correlation-stability coefficient (CS-coefficient). Values $>0.5$ indicate strong stability and interpretability (Epskamp et al., 2018).

Once the network was estimated on the estimation sample $(n=1010)$, results were mirrored on the replication sample $(n=1011)$ and both networks were compared using the network comparison test (van Borkulo et al., 2017) (5000 times repeated subsampling). We analyzed the network structure invariance, regarding to the differences in the connections of the edges of both networks (statistic $M)$; and the global strength invariance, based on the difference in global strength (the weighted absolute sum of all edges) between the two networks (statistic $S$ ). Additionally, if the omnibus network invariance test is significant, the test of edge invariance will also be estimated (van Borkulo et al., 2017).

The $\mathrm{R}$ packages used were: mvn version 5.8 (Korkmaz et al., 2014) to calculate the Mardia coefficient; bootnet version 1.3 (Epskamp et al., 2018) to perform the simulation analyses, to estimate the network, network stability and accuracy, and the strength of each node; igraph version 1.2.5 (Csardi \& Nepusz, 2006) to perform the modularity analysis; networktools version 1.2.2 (Jones, 2020) to estimate EI and bridge centrality indices; mgm version 1.2.7 (Haslbeck \& Waldorp, 2016) to estimate the explained variance ratio of each node; and NetworkComparisonTest 2.2.1 (van Borkulo et al., 2017) to test network invariance. All analyses were conducted using $\mathrm{R}$ version 3.6.3 and $\mathrm{R}-$ Studio version 1.2.5033.

\section{Results}

The descriptive statistics for each of the IDAS-II scales are shown in Table 2. The Cronbach's alpha coefficients were higher than 0.70 for all the IDAS-II scales and above 0.80 for 13 of the 18 scales.

\section{Network Estimation and Community Detection}

Table 3 shows the partial correlations and zero-order correlations between symptoms on estimation sample $(n=1010)$. The number of estimated edges with a partial correlation value that differed from zero was 93 out of 153 possible edges. The edge weights ranged from -0.46 (Euphoria-Low Well-Being) to 0.30 (Ordering - Checking, and Cleaning - Claustrophobia). Online Resource 2 shows the partial edge correlations estimated by bootstrapping with a $95 \% \mathrm{CI}$. 
Table 2 Results of the analysis of the scales and reliability of the IDAS-II $(n=2021)$

\begin{tabular}{lllrl}
\hline IDAS-II Scales & M (SD) & Skewness & Kurtosis & Coefficient $\alpha$ \\
\hline Appetite Gain & $5.87(2.87)$ & 1.17 & 0.84 & 0.78 \\
Appetite Loss & $4.85(2.51)$ & 1.48 & 1.76 & 0.86 \\
Checking & $5.76(2.72)$ & 1.10 & 0.71 & 0.77 \\
Claustrophobia & $8.03(4.36)$ & 1.64 & 2.15 & 0.87 \\
Cleaning & $11.51(5.12)$ & 0.95 & 0.57 & 0.85 \\
Dysphoria & $21.43(8.81)$ & 0.81 & -0.06 & 0.91 \\
Euphoria & $8.44(3.53)$ & 1.30 & 1.47 & 0.76 \\
Ill Temper & $9.77(4.54)$ & 1.08 & 0.59 & 0.86 \\
Insomnia & $13.18(6.11)$ & 0.81 & -0.18 & 0.90 \\
Lassitude & $12.24(4.70)$ & 0.84 & 0.37 & 0.78 \\
Low Well-Being & $25.61(6.26)$ & 009 & -0.41 & 0.85 \\
Mania & $9.76(4.38)$ & 0.92 & 0.10 & 0.80 \\
Ordering & $10.05(3.95)$ & 0.82 & 0.36 & 0.75 \\
Panic & $13.33(6.57)$ & 1.56 & 1.85 & 0.91 \\
Social Anxiety & $10.42(4.84)$ & 1.38 & 1.58 & 0.83 \\
Suicidality & $7.72(3.55)$ & 3.09 & 10.68 & 0.84 \\
Traumatic Avoid- & $8.50(4.02)$ & 0.73 & -0.27 & 0.84 \\
$\quad$ ance & & & & \\
Traumatic Intru- & $6.93(3.87)$ & 1.48 & 1.46 & 0.88 \\
sions & & & & \\
\hline
\end{tabular}

The estimated network in the estimation sample is shown in Fig. 1. Exploratory graphic analysis of modularity yielded an optimal solution of two communities. The first community includes the symptoms Dysphoria, Lassitude, Insomnia,
Suicidality, Appetite Loss, Appetite Gain, Ill Temper, Panic, Social Anxiety, and Traumatic Intrusions, while the second community includes Low Well-Being, Mania, Euphoria, Claustrophobia, Traumatic Avoidance, Checking, Ordering, and Cleaning. Figure 1 also shows the bridge nodes between both communities, which are Low Well-Being, Mania, Panic, and Claustrophobia.

\section{Network Inference}

The modularity index of this grouping was 0.25 while that obtained with the confirmatory factor analysis (CFA) presented in De la Rosa-Cáceres et al. (2020), based on the organization of the internal structure of IDAS-II and its correspondence with the Hierarchical Taxonomy of Psychopathology (HiTOP) model, was -0.06 (the latter network is presented in Online Resource 3).

The standardized scores estimated for the centrality indices of the nodes are shown in Table 4. The symptoms or nodes that present higher values of Strength, EI1, and EI2 are Dysphoria, Panic, and Mania (standardized values of Strength were between 2.95 and -1.01; standardized values of EI1 were between 2.26 and -2.31 ; and standardized values of EI2 were between 2.21 and -2.18).

CS-coefficient (cor $=0.7$ ) was 0.75 for Strength and EI (see Online Resource 4 and Online Resource 5,

Table 3 Partial and zero-order correlations between network symptoms on estimation sample $(n=1010)$

\begin{tabular}{|c|c|c|c|c|c|c|c|c|c|c|c|c|c|c|c|c|c|c|}
\hline Nodes & 1 & 2 & 3 & 4 & 5 & 6 & 7 & 8 & 9 & 10 & 11 & 12 & 13 & 14 & 15 & 16 & 17 & 18 \\
\hline 1. Appetite Gain & & 0.09 & 0.36 & 0.19 & 0.13 & 0.44 & 0.29 & 0.46 & 0.31 & 0.44 & 0.02 & 0.40 & 0.20 & 0.33 & 0.36 & 0.24 & 0.23 & 0.34 \\
\hline 2. Appetite Loss & -0.14 & & 0.31 & 0.38 & 0.15 & 0.58 & 0.18 & 0.42 & 0.52 & 0.47 & 0.17 & 0.39 & 0.17 & 0.55 & 0.44 & 0.40 & 0.36 & 0.46 \\
\hline 3. Checking & 0.05 & - & & 0.46 & 0.37 & 0.51 & 0.48 & 0.47 & 0.40 & 0.43 & -0.04 & 0.61 & 0.57 & 0.50 & 0.48 & 0.36 & 0.43 & 0.49 \\
\hline 4. Claustrophobia & -0.03 & 0.03 & 0.07 & & 0.44 & 0.52 & 0.31 & 0.43 & 0.44 & 0.40 & 0.10 & 0.51 & 34 & 0.61 & 0.56 & 0.48 & 0.42 & 0.47 \\
\hline Cle & - & - & 0.04 & 0.30 & & 0.18 & 0.25 & 0.18 & 0.20 & 0.18 & -0.08 & 0.29 & 0.42 & 0.22 & 0.22 & 0.15 & 0.30 & $0.1^{\top}$ \\
\hline 5. Dysp & 0.08 & 0.15 & 0.03 & - & 0.03 & & 0.25 & 0.73 & 0.69 & 0.74 & 0.35 & 0.65 & 0.27 & 0.81 & 0.72 & 0.61 & .48 & 0.77 \\
\hline 7. $\mathrm{E}$ & 0.06 & - & 0.07 & 0.01 & - & - & & 0.35 & 0.22 & 0.28 & -0.42 & 0.54 & 0.49 & 0.33 & 0.28 & 0.24 & 0.40 & 0.29 \\
\hline 3. Ill & 0.12 & - & - & - & - & 0.19 & 0.04 & & 0.56 & 0.62 & 0.17 & 0.62 & 0.28 & 0.68 & 0.60 & 0.53 & 0.38 & 0.6 \\
\hline Inst & 0.01 & 0.13 & - & 0.02 & - & 0.20 & - & 0.08 & & 0.53 & 0.22 & 0.52 & 0.24 & 0.64 & 0.54 & 0.46 & 0.39 & 0.62 \\
\hline 10. Las & 0.17 & 0.07 & - & - & - & 0.26 & - & 0.06 & 0.01 & & 0.18 & 0.59 & 0.24 & 0.65 & 0.56 & 0.44 & 0.39 & 0.58 \\
\hline 11. L & - & - & - & - & -0.01 & 0.18 & -0.46 & - & - & - & & 0.00 & -0.23 & 0.26 & 0.22 & 0.26 & -0.02 & 0.21 \\
\hline 12. Mania & 0.04 & - & 0.20 & 0.05 & - & 0.04 & 0.25 & 0.12 & 0.02 & 0.10 & - & & 0.49 & 0.67 & 0.55 & 0.46 & 0.50 & 0.58 \\
\hline 13. Ordering & - & - & 0.30 & - & 0.21 & - & 0.14 & - & - & -0.01 & -0.11 & 0.10 & & 0.27 & 0.29 & 0.20 & 0.39 & 0.27 \\
\hline 14. Panic & - & 0.13 & - & 0.13 & 0.01 & 0.19 & 0.02 & 0.07 & 0.10 & 0.15 & 0.07 & 0.17 & - & & 0.68 & 0.66 & 0.45 & 0.73 \\
\hline 15. Social Anxiety & 0.05 & - & 0.09 & 0.19 & - & 0.20 & - & 0.05 & - & 0.01 & 0.02 & 0.02 & - & 0.06 & & 0.56 & 0.41 & 0.6 \\
\hline 16. Suicidality & - & - & - & 0.05 & - & 0.03 & 0.02 & 0.08 & - & - & 0.11 & - & - & 0.17 & 0.12 & & 0.31 & 0.6 \\
\hline 17. Trauma. A & - & 0.07 & 0.03 & 0.10 & 0.06 & 0.05 & 0.12 & - & - & - & - & 0.08 & 0.09 & - & - & - & & .4 \\
\hline 18. Trauma. Intrusions & - & - & 0.06 & - & -0.02 & 0.19 & - & 0.07 & 0.11 & 0.01 & - & 0.02 & - & 0.12 & 0.09 & 0.16 & 0.16 & \\
\hline
\end{tabular}

Partial correlations are shown on the lower diagonal and zero-order correlations on the upper diagonal. The dashes represent correlation values $=0$ 


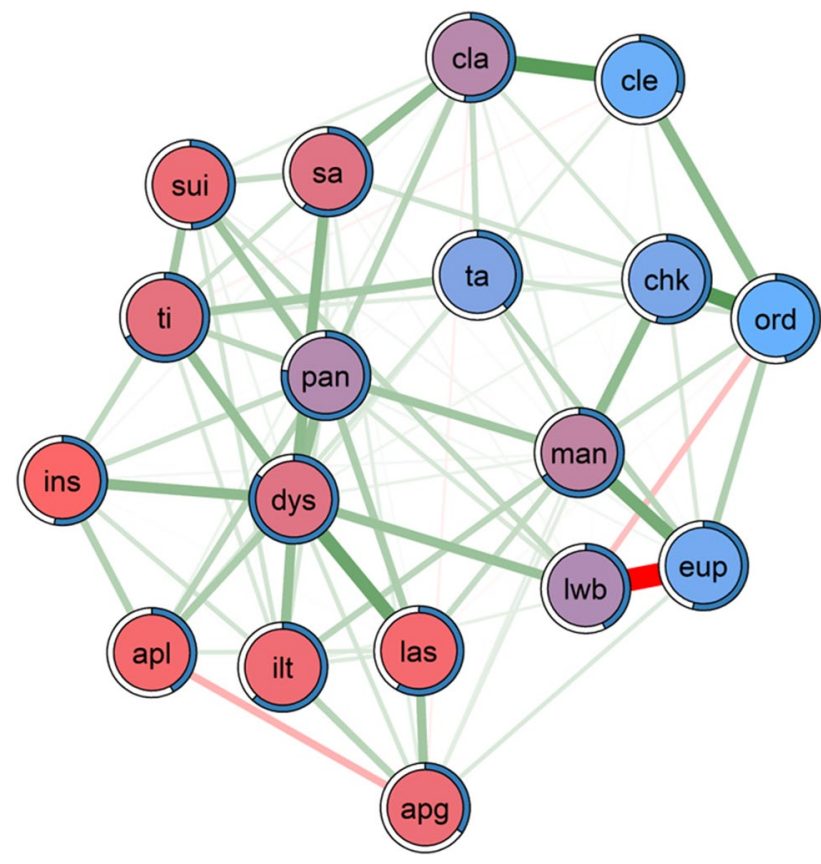

Fig. 1 Empirical network model (network structure estimated from a walktrap modularity analysis) apg: Appetite Gain, apl: Appetite Loss, chk: Checking, cla: Claustrophobia, cle: Cleaning, dys: Dysphoria, eup: Euphoria, ilt: Ill Temper, ins: Insomnia, las: Lassitude, lwb: Low Well-Being, man: Mania, ord: Ordering, pan: Panic, sa: Social Anxiety, sui: Suicidality, ta: Traumatic Avoidance, ti: Traumatic Intrusions. Each node represents a symptom. The edges represent the relationships (partial correlations) between the symptoms. Positive relationships are represented in green, and negative relationships in red. The thickness of the edge reflects the strength of the association, so that the most strongly related symptoms are connected by thicker edges. The blue pie chart surrounding each node represents the predictability of each node (a higher proportion of blue indicates greater predictability). The membership of the nodes to the different communities is represented by a red-blue gradient according to the bridge strength values: the symptoms of Community 1 are shown in red tones, the bridge symptoms in purple tones and the symptoms of Community 2 in blue tones. The arrangement of the nodes was established based on the Fruchterman-Reingold algorithm

respectively), indicating strong stability and interpretability of the estimates in both cases.

The standardized scores estimated for the bridge centrality indices of the nodes are shown in Table 4. The symptoms or nodes with higher values of Bridge Strength, Bridge EI1 and Bridge EI2 are Mania (standardized values of Bridge Strength $=2.04$; standardized values of Bridge EI1 = 2.09; and standardized values of Bridge EI2 = 2.39), Claustrophobia $(1.50,1.24$, and 1.09), Panic (1.14, 1.08, and 0.88), and Low Well-Being $(0.99,1.08$, and 1.02).

The predictability of each node is shown in Fig. 1 and Table 4 . The predictability values ranged from 0.30 (Cleaning) to 0.84 (Dysphoria), with a mean value of 0.54 . The symptoms with the least explained variance - and therefore the most independent - were Cleaning $\left(\mathrm{R}^{2}=0.30\right)$, Appetite Gain $\left(\mathrm{R}^{2}=0.35\right)$, Traumatic Avoidance $\left(\mathrm{R}^{2}=0.39\right)$ and Appetite Loss $\left(\mathrm{R}^{2}=0.42\right)$. The symptoms with the greatest explained variance were Dysphoria $\left(R^{2}=0.84\right)$, Panic $\left(R^{2}=0.77\right)$, Traumatic Intrussions $\left(R^{2}=0.67\right)$, and Mania $\left(\mathrm{R}^{2}=0.66\right)$.

The PC and PR values are shown in Table 4. The highest PC values corresponded to Traumatic Avoidance $(\mathrm{PC}=0.43)$, Social Anxiety $(\mathrm{PC}=0.39)$, and Claustrophobia $(\mathrm{PC}=0.35)$, these being the nodes that distribute their edges most equally among the network communities. The network nodes that presented the strongest and most numerous edges, according to $\mathrm{PR}$ values, were Dysphoria $(\mathrm{PR}=1)$, Panic $(P R=0.77)$, and Mania $(P R=0.60)$.

The invariance test showed that the networks estimated on estimation $(n=1010)$ and replication subsamples $(n=1011)$ did not differ significantly in structure (i.e., differences in the edge connections of the two networks, $M=0.08 ; p=0.989$ ) nor in overall strength (i.e., differences in the sum of the absolute weights between the two networks, $S=0.24$; $p=0.418$ ). The overall strength of the network estimated over the first half of the sample was 8.69 , while that of the network estimated over the second half of the sample was 8.45 .

\section{Discussion}

The present work examined the relationships between the dimensional symptoms assessed by the IDAS-II improving our understanding of comorbidity between emotional disorders. Results showed that emotional disorders symptoms groups in two communities: first linked to symptoms of depression and anxiety and second grouping symptoms of bipolar disorder and obsessive-compulsive disorder. Mania, Panic, Claustrophobia, and Low Well-Being emerged as bridge symptoms, connecting the two substructures. Finally, results suggest that Dysphoria is the symptom with greatest number of connections with rest of depression dimensions; Traumatic Intrusions the one with most conecctions for PTSD, and Checking and Ordering for OCD. The invariance analysis failed to find significant differences between the structure and global strength of estimation and replication subsample networks. Next, we analyze the results in more detail.

Congruent with our first hypothesis, the structure of the network provides evidence of a stronger relationship between the symptoms of depression and anxiety (Dysphoria, Lassitude, Insomnia, Suicidality, Appetite Loss, Appetite Gain, Ill Temper, Panic and Social Anxiety) that are grouped in the first community. This substructure, previously described (Bekhuis et al., 2016; McElroy et al., 2018) supports the observed relationship between these two sets of disorders. Based on this body of evidence, various authors 
Table 4 Standardized values of centrality indices and bridge centrality indices, explained variance, Participation Coefficient, and Participation Ratio of each node on estimation sample $(n=1010)$

\begin{tabular}{|c|c|c|c|c|c|c|c|c|c|}
\hline Nodes & Strength & $\begin{array}{l}\text { Expected } \\
\text { Influence } 1\end{array}$ & $\begin{array}{l}\text { Expected } \\
\text { Influence } 2\end{array}$ & Bridge Strength & $\begin{array}{l}\text { Bridge } \\
\text { Expected Influ- } \\
\text { ence } 1\end{array}$ & $\begin{array}{l}\text { Bridge } \\
\text { Expected Influ- } \\
\text { ence } 2\end{array}$ & $\mathrm{R}^{2}$ & $\mathrm{PC}$ & PR \\
\hline Appetite Gain & -0.77 & -0.85 & -1.02 & -0.33 & -0.50 & -0.73 & 0.35 & 0.11 & 0.15 \\
\hline Appetite Loss & -0.87 & -0.80 & -0.77 & -0.89 & -0.70 & -0.78 & 0.42 & 0.07 & 0.03 \\
\hline Checking & -0.12 & 0.35 & 0.21 & -0.06 & 0.09 & 0.28 & 0.54 & 0.09 & 0.32 \\
\hline Claustrophobia & 0.04 & 0.34 & 0.18 & 1.50 & 1.24 & 1.09 & 0.52 & 0.35 & 0.35 \\
\hline Cleaning & -1.01 & -0.59 & -0.91 & -1.17 & -1.75 & -1.62 & 0.30 & 0.01 & 0 \\
\hline Dysphoria & 2.95 & 2.26 & 2.21 & 0.62 & 0.36 & 0.13 & 0.84 & 0.12 & 1 \\
\hline Euphoria & 0.79 & -1.19 & -0.79 & -0.57 & -0.39 & -0.76 & 0.54 & 0.02 & 0.42 \\
\hline Ill Temper & -0.29 & 0.24 & 0.33 & -0.48 & -0.30 & -0.17 & 0.62 & 0.17 & 0.29 \\
\hline Insomnia & -0.97 & -0.22 & -0.14 & -1.29 & -1.08 & -0.86 & 0.53 & 0.01 & 0.11 \\
\hline Lassitude & -0.39 & 0.11 & 0.32 & -0.80 & -0.77 & -0.46 & 0.58 & 0.08 & 0.22 \\
\hline Low Well-Being & -0.02 & -2.31 & -2.18 & 0.99 & 1.08 & 1.02 & 0.43 & 0.18 & 0.10 \\
\hline Mania & 0.80 & 0.96 & 0.88 & 2.04 & 2.09 & 2.39 & 0.66 & 0.33 & 0.60 \\
\hline Ordering & 0.00 & -0.17 & -0.35 & -1.48 & -1.46 & -1.34 & 0.46 & 0.00 & 0.17 \\
\hline Panic & 1.48 & 1.36 & 1.54 & 1.14 & 1.08 & 0.88 & 0.77 & 0.26 & 0.77 \\
\hline Social Anxiety & -0.28 & 0.24 & 0.40 & 0.58 & 0.70 & 0.64 & 0.60 & 0.39 & 0.35 \\
\hline Suicidality & -0.80 & -0.10 & -0.27 & -0.32 & -0.16 & -0.51 & 0.48 & 0.17 & 0.04 \\
\hline Traumatic Avoidance & -0.72 & 0.06 & -0.29 & 0.34 & 0.47 & 0.60 & 0.39 & 0.43 & 0.16 \\
\hline Traumatic Intrusions & 0.17 & 0.43 & 0.63 & 0.18 & 0.18 & 0.21 & 0.67 & 0.22 & 0.38 \\
\hline
\end{tabular}

$R^{2}=$ explained variance; $P C=$ Participation Coefficient $P R=$ Participation Ratio

have questioned whether these disorders should be defined as a single clinical entity and not as distinct disorders (Borsboom, 2017; Kotov et al., 2017; McElroy et al., 2018).

The structure of the network also shows, in a separate community, the symptoms of BD (Euphoria and Mania) and OCD (Checking, Ordering, and Cleaning). The cooccurrence of these disorders has been reported in several studies and meta-analyses (e.g., Ferentinos et al., 2020). The grouping of this set of symptoms is of interest, as Tonna et al. (2016) point out that the appearance of OCD symptoms could be a risk factor for increased BD symptoms.

Interestingly, the two PTSD symptom dimensions appear separately. In line with Gilbar (2020) and Levi-Belz et al. (2020), Traumatic Intrusions appear in Community 1, linked to symptoms of anxiety and depression, whilst Traumatic Avoidance is linked to OCD symptoms. This evidence supports the connection between PTSD and depressive disorder (Afzali et al., 2017; Levi-Belz et al., 2020) through intrusive thoughts. In contrast, strategies to reduce anxiety had the most direct connection with OCD. OCD and PTSD share avoidance of stimuli as a means of reducing stress (American Psychiatric Association, 2013; Franklin \& Raines, 2019). From a behavioral perspective, Gershuny et al. (2003) point out that, in patients with comorbid OCD and PTSD, the avoidance strategies in OCD could reduce the emotional distress generated by a traumatic event, thus explaining the comorbidity of these disorders.
Concerning our second hypothesis, the results indicate that the bridge symptoms differ from those hypothesized. Although Ill Temper has been pointed out as a bridge symptom (Afzali et al., 2017; Choi et al., 2017), the connections observed in the present work between this symptom and PTSD occur within the same community. In the present work, the bridge nodes obtained are Claustrophobia, Low Well-Being, Mania, and Panic. It can be observed that whilst Panic connects the symptoms of depression and anxiety, Mania has been shown to connect the symptoms of depression with BD. Niitsu et al. (2015) highlight panic attacks as one of the strongest predictors of the change from depression to mania in $\mathrm{BD}$. In relation to the other two bridge symptoms (Low Wellbeing and Claustrophobia), to our knowledge, this is the first work that includes these dimensions in network analysis. The inclusion of a greater number of symptoms can modify community organization and, therefore, the identification of bridge symptoms (Jones et al., 2019). For Claustrophobia, the observed relationships with Panic and Social Anxiety provide further empirical support for the organization proposed by HiTOP (Kotov et al., 2017) where these symptoms are grouped within the Fear factor. With regard to Low Well-Being, differential activation is observed in the network structure, with activation 
of Community 1 and inhibition of Community 2. For several decades, numerous studies have provided evidence for the relationship between Low Well-Being and depression (Kotov et al., 2017; Watson et al., 2007).

Finally, regarding the symptoms with the greatest influence, our results support the third hypothesis. Similar to Funkhouser et al. (2020), Dysphoria presents the highest centrality values for depression. Likewise, Traumatic Intrusions showed greater centrality than did Traumatic Avoidance for PTSD (Contractor et al., 2020; Gilbar, 2020), and Checking and Ordering showed greater centrality for OCD (Zhang et al., 2019). Although Dysphoria is the most central node of the network, the low PC values and location indicate that their edges are primarily related to Community 1 . This should be regarded as an important symptom in the initiation and maintenance of depression and anxiety disorders (Boschloo et al., 2016).

In clinical terms, some authors suggest that clinicians should pay attention to the presence of bridging symptoms and intervene on them to prevent comorbidity (Fried et al., 2017; Jones et al., 2019; Kaiser et al., 2021). In this sense, the intervention on panic and claustrophobia-identified as bridge symptoms on present study -, supports the inclusion of some of the modules of the Unified Protocol for Transdiagnostic Treatment of Emotional Disorders (UP) Barlow et al., 2017), successfully applied to a wide range of disorders. Specifically, both modules 5-aimed on changing the action tendencies related to strong emotions, and 7 - emotion exposures -, appear as central modules of the UP. Related to this, Jones et al. (2019) suggest that deactivation of bridging symptoms could prevent comorbidity to a greater extent than the deactivation of more central symptoms in the network. Thus, Dysphoria could be considered as a target of intervention for depressive disorders, rather than a symptom wich intervention could prevent comorbidity.

\section{Conclusions}

From a clinical perspective, this study provides useful information. According to previous studies (Afzali et al., 2017; Borsboom, 2017; Jones et al., 2019), interventions that target bridge symptoms could be relevant due to their role in the activation of different communities. From the analysis of the heterogeneity, results suggest that interventions aimed at Panic, Mania, and Claustrophobia may generate more global changes because their edges are distributed more equally among the communities. Moreover, Panic and Mania have stronger and more numerous edges. In congruence with this result, a systematic review conducted by González-Robles et al. (2018) found that Panic is the symptom most frequently targeted in transdiagnostic treatments of emotional disorders. Treatment of these symptoms could help to reduce the severe consequences associated with comorbidities, such as greater functional impairment, higher rates of chronicity, and increased disability (Hofmeijer-Sevink et al., 2012).

Finally, several studies suggest that treatments applied to nodes with high values of strength, IE, and predictability could generate global changes in the structure of the network and could be considered as priority interventions (Haslbeck \& Waldorp, 2018; Robinaugh et al., 2016; Zhang et al., 2019). According to this idea, one of the priority targets of the network could be Dysphoria. This result is consistent with the notion that negative affect is the common component of emotional disorders related to the internalizing spectrum (Kotov et al., 2017). However, Santos et al. (2017) point out the need to also intervene in other less central symptoms when they cause clinically significant discomfort.

Although this study makes several contributions to the literature, it is not without limitations. First, the use of a transversal design does not allow for making causal inferences about the direction of associations between symptoms, which was the reason for using undirected networks in present study. In this regard, it would be worthwhile to conduct future longitudinal studies to clarify the directionality of the associations in the network.

Second the resulting IDAS-II network does not include other symptoms of depression such as concentration or psychomotor problems which, although not diagnostic criteria of the DSM, could be equally relevant (Fried et al., 2016). As mentioned above, the inclusion of different symptoms can produce changes on the community organization and, therefore, the identification of bridge symptoms (Jones et al., 2019). However, it should be mentioned that this is one of the studies evaluating the greatest number of internalizing symptoms within the same instrument, using a dimensional approach.

Finally, it should be noted that different sampling procedures have been used to select the subsamples: community adults were recruited by random sampling, while, patients were selected by non-probability sampling. The use of nonprobabilistic sampling may limit the generalizability of patient responses, though the accessibility of this kind of patients difficult the use of random sampling procedures.

In general terms, we believe the present study contributes providing relevant information for a better understanding of emotional disorders comorbidities, one of the most persistent problems associated with mental disorders (Cramer et al., 2010; Hofmeijer-Sevink et al., 2012; Kotov et al., 2017), and also for the development of transdiagnostic interventions for various emotional disorders by identifying the core symptoms within the internalizing spectrum (Barlow et al., 2017).

Supplementary Information The online version contains supplementary material available at https://doi.org/10.1007/s12144-022-02907-4. 
Authors' Contributions $\mathrm{CDB}, \mathrm{FFC}$ and $\mathrm{ADC}$ has contributed on the conception and design of study. ADC, FFC and MSG has contributed on the acquisition of data. ADC, MSG and PSJ had participated on the analysis of data. ADC, SSO, CDB had participated on the interpretation of data. ADC, CDB, SSO had drafted the manuscript. All authors have revised the manuscript critically for intellectual content and approved the final version of the manuscript submitted.

Funding Open Access funding provided thanks to the University of Huelva / CRUE-CSIC agreement with Springer Nature. This work was supported by the grant "Network-Psyco: Modelización a través de redes empíricas de las conexiones entre facetas y rasgos psicológicos", project UHU-1257470 on Programa Operativo FEDER Andalucía 2014-2020, provided by Fondo Europeo de Desarrollo Regional (EU) and Junta de Andalucía (Spain), and by Ministry of Universities of the Government of Spain (FPU19/00144).

Data Availability The datasets analysed during the current study are available in Arias Montano, the Institutional Repository of the University of Huelva, http://hdl.handle.net/10272/20199

\section{Declarations}

Ethics Approval This study was approved by the Bioethics Committee of Biomedical Research of Andalusia (Spain) (No. PI 040/18). The procedures used in this study adhere to the tenets of the Declaration of Helsinki.

Consent to Participate Informed consent was obtained from all individual participants included in the study.

Informed Consent Informed consent was obtained from all individual participants included in the study.

Competing Interests The authors have no relevant financial or nonfinancial interests to disclose.

Research Involving Human Participants and/or Animals This study was approved by the Bioethics Committee of Biomedical Research of Andalusia (Spain) (No. PI 040/18). The procedures used in this study adhere to the tenets of the Declaration of Helsinki.

Open Access This article is licensed under a Creative Commons Attribution 4.0 International License, which permits use, sharing, adaptation, distribution and reproduction in any medium or format, as long as you give appropriate credit to the original author(s) and the source, provide a link to the Creative Commons licence, and indicate if changes were made. The images or other third party material in this article are included in the article's Creative Commons licence, unless indicated otherwise in a credit line to the material. If material is not included in the article's Creative Commons licence and your intended use is not permitted by statutory regulation or exceeds the permitted use, you will need to obtain permission directly from the copyright holder. To view a copy of this licence, visit http://creativecommons.org/licenses/by/4.0/.

\section{References}

Afzali, M. H., Sunderland, M., Teesson, M., Carragher, N., Mills, K., \& Slade, T. (2017). A network approach to the comorbidity between posttraumatic stress disorder and major depressive disorder: The role of overlapping symptoms. Journal of Affective Disorders, 208, 490-496. https://doi.org/10.1016/j.jad.2016.10.037
American Psychiatric Association. (2013). Diagnostic and statistical manual of mental disorders (5th ed.). Washington, DC: Author.

Barlow, D. H., Farchione, T. J., Bullis, J. R., Gallagher, M. W., MurrayLatin, H., Sauer-Zavala, S., Bentley, K. H., Thompson-Hollands, J., Conklin, L. R., Boswell, J. F., Ametaj, A., Carl, J. R., Boettcher, H. T., \& Cassiello-Robbins, C. (2017). The unified protocol for transdiagnostic treatment of emotional disorders compared with diagnosis-specific protocols for anxiety disorders: A randomized clinical trial. JAMA Psychiatry, 74(9), 875-884. https://doi.org/ 10.1001/jamapsychiatry.2017.2164

Bekhuis, E., Schoevers, R. A., Van Borkulo, C. D., Rosmalen, J. G. M., $\&$ Boschloo, L. (2016). The network structure of major depressive disorder, generalized anxiety disorder and somatic symptomatology. Psychological Medicine, 46(14), 2989-2998. https://doi.org/ 10.1017/S0033291716001550

Borsboom, D. (2017). A network theory of mental disorders. World Psychiatry, 16(1), 5-13. https://doi.org/10.1002/wps.20375

Boschloo, L., Van Borkulo, C. D., Borsboom, D., \& Schoevers, R. A. (2016). A prospective study on how symptoms in a network predict the onset of depression. Psychotherapy and Psychosomatics, 85(3), 183-184. https://doi.org/10.1159/000442001

Chen, J., \& Chen, Z. (2008). Extended bayesian information criteria for model selection with large model spaces. Biometrika, 95(3), 759-771. https://doi.org/10.1093/biomet/asnO34

Choi, K. W., Batchelder, A. W., Ehlinger, P. P., Safren, S. A., \& O'Cleirigh, C. (2017). Applying network analysis to psychological comorbidity and health behavior: Depression, PTSD, and sexual risk in sexual minority men with trauma histories. Journal of Consulting and Clinical Psychology, 85(12), 1158-1170. https:// doi.org/10.1037/ccp0000241

Contractor, A. A., Greene, T., Dolan, M., Weiss, N. H., \& Armour, C. (2020). Relation between PTSD symptom clusters and positive memory characteristics: A network perspective. Journal of Anxiety Disorders, 69, 102157. https://doi.org/10.1016/j.janxdis. 2019.102157

Contreras, A., Nieto, I., Valiente, C., Espinosa, R., \& Vazquez, C. (2019). The study of psychopathology from the network analysis perspective: A systematic review. Psychotherapy and Psychosomatics, 88(2), 71-83. https://doi.org/10.1159/000497425

Cramer, A. O. J., Waldorp, L. J., Van Der Maas, H. L. J., \& Borsboom, D. (2010). Comorbidity: A network perspective. Behavioral and Brain Sciences, 33(2-3), 137-150. https://doi.org/10.1017/S0140 525X09991567

Csardi, G., \& Nepusz, T. (2006). The igraph software package for complex network research. InterJournal, Complex Systems 1695. http://igraph.org

De Berardis, D., Fornaro, M., Valchera, A., Cavuto, M., Perna, G., Di Nicola, M., Serafini, G., Carano, A., Pompili, M., Vellante, F., Orsolini, L., Fiengo, A., Ventriglio, A., Yong-Ku, K., Martinotti, G., Di Giannantonio, M., \& Tomasetti, C. (2018). Eradicating suicide at its roots: Preclinical bases and clinical evidence of the efficacy of ketamine in the treatment of suicidal behaviors. International Journal of Molecular Sciences, 19(10). https://doi.org/ 10.3390/ijms 19102888

De la Rosa-Cáceres, A., Stasik-O’Brien, S. M., Rojas, A. J., SanchezGarcía, M., Lozano, O. M., \& Díaz-Batanero, C. (2020). Spanish adaptation of the Inventory of Depression and Anxiety Symptoms (IDAS-II) and a study of its psychometric properties. Journal of Affective Disorders, 271, 81-90. https://doi.org/10.1016/j.jad. 2020.03.187

Epskamp, S., Borsboom, D., \& Fried, E. I. (2018). Estimating psychological networks and their accuracy: A tutorial paper. Behavior Research Methods, 50(1), 195-212. https://doi.org/10.3758/ s13428-017-0862-1

Ferentinos, P., Preti, A., Veroniki, A. A., Pitsalidis, K. G., Theofilidis, A. T., Antoniou, A., \& Fountoulakis, K. N. (2020). Comorbidity 
of obsessive-compulsive disorder in bipolar spectrum disorders: Systematic review and meta-analysis of its prevalence. Journal of Affective Disorders, 263, 193-208. https://doi.org/10.1016/j. jad.2019.11.136

Franklin, C. L., \& Raines, A. M. (2019). The overlap between OCD and PTSD: Examining self-reported symptom differentiation. Psychiatry Research, 280(112508). https://doi.org/10.1016/j. psychres.2019.112508

Fried, E. I., Epskamp, S., Nesse, R. M., Tuerlinckx, F., \& Borsboom, D. (2016). What are "good" depression symptoms? Comparing the centrality of DSM and non-DSM symptoms of depression in a network analysis. Journal of Affective Disorders, 189, 314-320. https://doi.org/10.1016/j.jad.2015.09.005

Fried, E. I., van Borkulo, C. D., Cramer, A. O. J., Boschloo, L., Schoevers, R. A., \& Borsboom, D. (2017). Mental disorders as networks of problems: a review of recent insights. Social Psychiatry and Psychiatric Epidemiology, 52(1). https://doi.org/ 10.1007/s00127-016-1319-z

Friedman, J., Hastie, T., \& Tibshirani, R. (2008). Sparse inverse covariance estimation with the graphical lasso. Biostatistics, 9(3), 432-441. https://doi.org/10.1093/biostatistics/kxm045

Fruchterman, T. M. J., \& Reingold, E. M. (1991). Graph drawing by force-directed placement. Software: Practice and Experience, 21(11), 1129-1164. https://doi.org/10.1002/spe.4380211102

Funkhouser, C. J., Correa, K. A., Gorka, S. M., Nelson, B. D., Phan, K. L., \& Shankman, S. A. (2020). The replicability and generalizability of internalizing symptom networks across five samples. Journal of Abnormal Psychology, 129(2), 191-203. https://doi. org/10.1037/abn0000496

Gershuny, B. S., Baer, L., Radomsky, A. S., Wilson, K. A., \& Jenike, M. A. (2003). Connections among symptoms of obsessive-compulsive disorder and posttraumatic stress disorder: A case series. Behaviour Research and Therapy, 41(9), 1029-1041. https:// doi.org/10.1016/S0005-7967(02)00178-X

Gilbar, O. (2020). Examining the boundaries between ICD-11 PTSD/ CPTSD and depression and anxiety symptoms: A network analysis perspective. Journal of Affective Disorders, 262, 429-439. https://doi.org/10.1016/j.jad.2019.11.060

González-Robles, A., Díaz-García, A., Miguel, C., García-Palacios, A., \& Botella, C. (2018). Comorbidity and diagnosis distribution in transdiagnostic treatments for emotional disorders: A systematic review of randomized controlled trials. PLOS ONE, 13(11), e0207396. https://doi.org/10.1371/journal.pone.02073 96

Haslbeck, J. M. B., \& Waldorp, L. J. (2016). mgm: Estimating timevarying mixed graphical models in high-dimensional data. $V V($ Ii). http://arxiv.org/abs/1510.06871

Haslbeck, J. M. B., \& Waldorp, L. J. (2018). How well do network models predict observations? On the importance of predictability in network models. Behavior Research Methods, 50(2), 853-861. https://doi.org/10.3758/s13428-017-0910-x

Hofmeijer-Sevink, M. K., Batelaan, N. M., Van Megen, H. J. G. M., Penninx, B. W., Cath, D. C., Van Den Hout, M. A., \& Van Balkom, A. J. L. M. (2012). Clinical relevance of comorbidity in anxiety disorders: A report from the Netherlands Study of Depression and Anxiety (NESDA). Journal of Affective Disorders, 137(1-3), 106-112. https://doi.org/10.1016/j.jad.2011. 12.008

Jones, P. (2020). Networktools: Tools for Identifying Important Nodes in Networks. R package version 1.2.2. https://cran.r-proje ct.org/package $=$ networktools

Jones, P., Ma, R., \& McNally, R. J. (2019). Bridge Centrality: A network approach to understanding comorbidity. Multivariate Behavioral Research, 56(2), 353-367. https://doi.org/10.1080/ 00273171.2019 .1614898
Kaiser, T., Herzog, P., Voderholzer, U., \& Brakemeier, E. L. (2021). Unraveling the comorbidity of depression and anxiety in a large inpatient sample: Network analysis to examine bridge symptoms. Depression and Anxiety, August 2020, 1-11. https://doi. org/10.1002/da.23136

Korkmaz, S., Goksuluk, D., \& Zararsiz, G. (2014). MVN: An R package for assessing multivariate normality. $R$ Journal, 6(2), 151-162. https://doi.org/10.32614/rj-2014-031

Kotov, R., Waszczuk, M. A., Krueger, R. F., Forbes, M. K., Watson, D., Clark, L. A., Achenbach, T. M., Althoff, R. R., Ivanova, M. Y., Michael Bagby, R., Brown, T. A., Carpenter, W. T., Caspi, A., Moffitt, T. E., Eaton, N. R., Forbush, K. T., Goldberg, D., Hasin, D., Hyman, S. E., ... Zimmerman, M. (2017). The Hierarchical Taxonomy of Psychopathology (HiTOP): A dimensional alternative to traditional nosologies. Journal of Abnormal Psychology, 126(4), 454-477. https://doi.org/10. 1037/abn0000258

Letina, S., Blanken, T. F., Deserno, M. K., \& Borsboom, D. (2019). Expanding network analysis tools in psychological networks: Minimal spanning trees, participation coefficients, and motif analysis applied to a network of 26 psychological attributes. Complexity, 2019, 9424605. https://doi.org/10.1155/2019/ 9424605

Levi-Belz, Y., Greene, T., \& Zerach, G. (2020). Associations between moral injury, PTSD clusters, and depression among Israeli veterans: A network approach. European Journal of Psychotraumatology, 11(1), 1736411. https://doi.org/10.1080/ 20008198.2020.1736411

Liu, H., Lafferty, J., \& Wasserman, L. (2009). The nonparanormal: Semiparametric estimation of high dimensional undirected graphs. Journal of Machine Learning Research, 10, 2295-2328. https://doi.org/10.1184/r1/6610712

McElroy, E., Fearon, P., Belsky, J., Fonagy, P., \& Patalay, P. (2018). Networks of depression and anxiety symptoms across development. Journal of the American Academy of Child and Adolescent Psychiatry, 57(12), 964-973. https://doi.org/10.1016/j.jaac. 2018.05.027

McNally, R. J., Mair, P., Mugno, B. L., \& Riemann, B. C. (2017). Co-morbid obsessive-compulsive disorder and depression: A Bayesian network approach. Psychological Medicine, 47(7), 1204-1214. https://doi.org/10.1017/S0033291716003287

Niitsu, T., Fabbri, C., \& Serretti, A. (2015). Predictors of switch from depression to mania in bipolar disorder. Journal of Psychiatric Research, 66-67, 45-53. https://doi.org/10.1016/j.jpsychires. 2015.04.014

Orsolini, L., Latini, R., Pompili, M., Serafini, G., Volpe, U., Vellante, F., Fornaro, M., Valchera, A., Tomasetti, C., Fraticelli, S., Alessandrini, M., La Rovere, R., Trotta, S., Martinotti, G., Di Giannantonio, M., \& De Berardis, D. (2020). Understanding the complex of suicide in depression: From research to clinics. Psychiatry Investigation, 17(3), 207-221. https://doi.org/10.30773/pi.2019. 0171

Pons, P., \& Latapy, M. (2005). Computing communities in large networks using random walks. In P. Yolum, T. Güngör, F. Gürgen, \& C. Özturan (Eds.), Computer and Information Sciences-ISCIS 2005 (pp. 284-293). Springer.

Quevedo, L. de A., Loret de Mola, C., Pearson, R., Murray, J., Hartwig, F. P., Gonçalves, H., Pinheiro, R. T., Gigante, D. P., Motta, J. V. dos S., Quadros, L. de C. M. de, Barros, F. C., \& Horta, B. L. (2020). Mental disorders, comorbidities, and suicidality at 30 years of age in a Brazilian birth cohort. Comprehensive Psychiatry, 102, 152194 Contents. https://doi.org/10.1016/j.comppsych. 2020.152194

Robinaugh, D. J., Millner, A. J., \& McNally, R. J. (2016). Identifying highly influential nodes in the complicated grief network. Journal 
of Abnormal Psychology, 125(6), 747-757. https://doi.org/10. 1037/abn0000181

Santos, H., Fried, E. I., Asafu-Adjei, J., \& Jeanne Ruiz, R. (2017). Network structure of perinatal depressive symptoms in latinas: Relationship to stress and reproductive biomarkers. Research in Nursing and Health, 40(3), 218-228. https://doi.org/10.1002/nur. 21784

Skodol, A. E. (2012). Personality disorders in DSM-5. Annual Review of Clinical Psychology, 8(1), 317-344. https://doi.org/10.1146/ annurev-clinpsy-032511-143131

Tonna, M., Amerio, A., Odone, A., Stubbs, B., \& Ghaemi, S. N. (2016). Comorbid bipolar disorder and obsessive-compulsive disorder: Which came first? Australian and New Zealand Journal of Psychiatry, 50(7), 695-698. https://doi.org/10.1177/00048 67415621395

van Borkulo, C. D., Boschloo, L., Kossakowski, J. J., Tio, P., Schoevers, R. A., Borsboom, D., \& Waldorp, L. J. (2017). Comparing network structures on three aspects: A permutation test. Manuscript Submitted, March, 34. https://doi.org/10.13140/RG.2.2. 29455.38569

Watson, D., O'Hara, M. W., Naragon-Gainey, K., Koffel, E., Chmielewski, M., Kotov, R., Stasik-O’Brien, S. M., \& Ruggero, C. J. (2012). Development and validation of new anxiety and bipolar symptom scales for an expanded version of the IDAS (the IDAS-II). Assessment, 19(4), 399-420. https://doi.org/10.1177/ 1073191112449857
Watson, D., O’Hara, M. W., Simms, L. J., Kotov, R., Chmielewski, M., McDade-Montez, E. A., Gamez, W., \& Stuart, S. (2007). Development and validation of the Inventory of Depression and Anxiety Symptoms (IDAS). Psychological Assessment, 19(3), 253-268. https://doi.org/10.1037/1040-3590.19.3.253

World Health Organization. (2017). Depression and other common mental disorders. Global Health estimates. World Health Organization. https://apps.who.int/iris/handle/10665/254610

Zhang, R. T., Zhou, H. Y., Wang, Y. M., Yang, Z. Y., Wang, Y., So, S. H., Chiu, C. D., Leung, P., Cheung, E., \& Chan, R. (2019). Network analysis of schizotypal personality traits and their association with other subclinical psychiatric features. Asian Journal of Psychiatry, 44, 209-216. https://doi.org/10.1016/j.ajp.2019. 08.005

Publisher's Note Springer Nature remains neutral with regard to jurisdictional claims in published maps and institutional affiliations. 